Musées, Patrimoine et Culture scientifiques et techniques

172 | 2017

juillet-août 2017

\title{
Étudier le comportement des visiteurs dans un musée d'histoire du Débarquement
}

Stanislas Hommet, Océane Costey and Maëva Hardelay

\section{OpenEdition \\ Journals}

Electronic version

URL: http://journals.openedition.org/ocim/1830

DOI: $10.4000 /$ ocim. 1830

ISSN: 2108-646X

\section{Publisher}

OCIM

Printed version

Date of publication: 1 July 2017

Number of pages: $22-27$

ISSN: 0994-1908

Electronic reference

Stanislas Hommet, Océane Costey and Maëva Hardelay, «Étudier le comportement des visiteurs dans un musée d'histoire du Débarquement », La Lettre de I'OCIM [Online], 172 | 2017, Online since 01 July 2018, connection on 01 May 2019. URL : http://journals.openedition.org/ocim/1830 ; DOI : 10.4000/ ocim. 1830

This text was automatically generated on 1 May 2019.

Tous droits réservés 


\section{Étudier le comportement des visiteurs dans un musée d'histoire du Débarquement}

Stanislas Hommet, Océane Costey and Maëva Hardelay

Le musée du Débarquement d'Arromanches

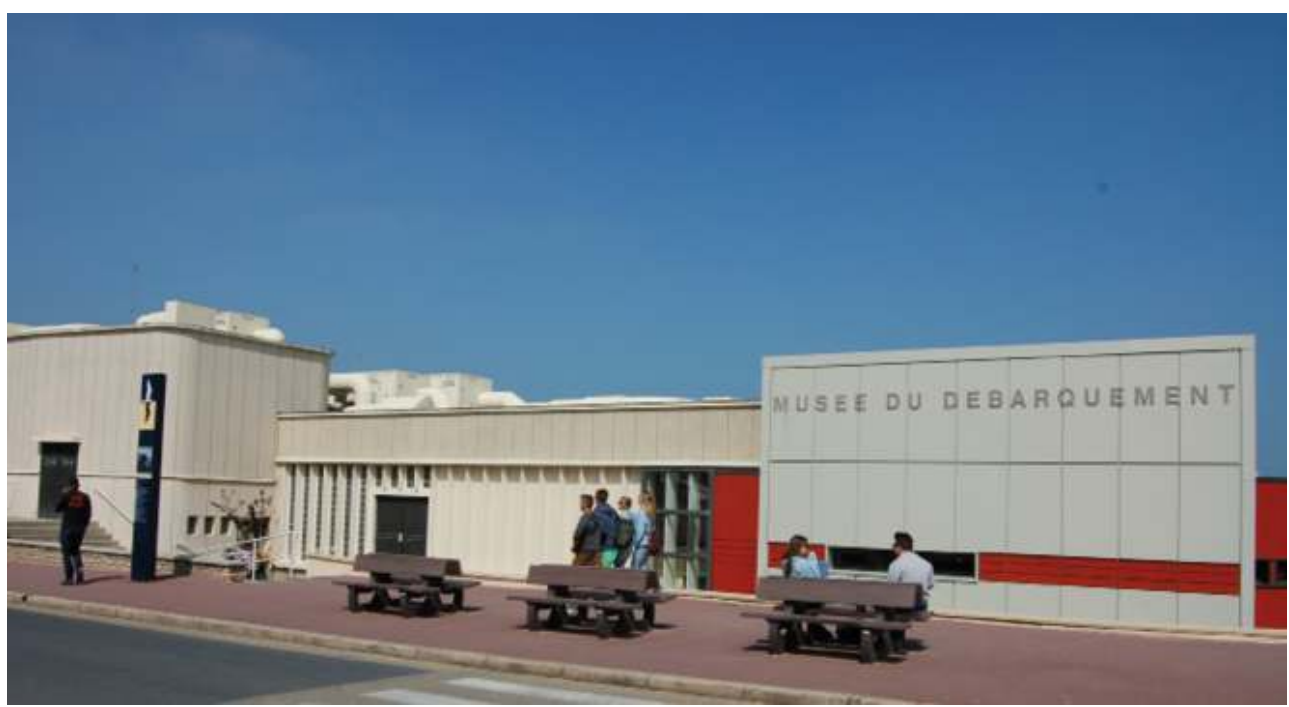

(c) S. Hommet

\section{L'origine d'une étude de réception}

La région Basse-Normandie a mis en place depuis deux ans une politique de classement des plages du débarquement. À ce titre, elle s'est tournée vers l'École Supérieure du Professorat et de l'Éducation (ESPE) de l'université de Caen-Normandie qui porte en son sein un parcours de formation de Master 2 en médiation culturelle, pour mener une série 
d'études de réception en espace muséal. Cet article présente les résultats de l'étude qui a fait l'objet d'un stage de trois mois de deux étudiantes en médiation culturelle Océane Costey et Maëva Hardelay, au musée du Débarquement d'Arromanches, qui ont pu mettre en œuvre, à cette occasion une étude qualitative de réception par les publics de l'exposition d'histoire. Cette présentation est un travail mené en formation par les deux étudiantes.

2 Évaluer ce que pensent les publics de la visite par des enquêtes de satisfaction est assez répandu mais ne permet en aucun cas d'aller au plus près des réalités des expériences de visites des visiteurs. Comment les visiteurs des musées réagissent-ils face aux œuvres exposées ? Car ce public qui se presse au musée du Débarquement d'Arromanches, qu'il soit familial, touristique qu'il soit individuel ou en groupe, n'est pas vierge d'expériences de visites. Il a sa propre représentation de l'espace pour lequel il est prêt à verser quelques euros, imaginant ou s'attendant à voir tel ou tel point d'explication. Il mène ses propres comparaisons, et surtout établit sa visite selon ses envies, besoins de découverte. Si le visiteur réalise sa visite avec ses pieds, rapidement le cerveau prend le dessus et impose tel ou tel déplacement.

Oui, le visiteur pense sa visite et souvent indépendamment de toute scénographie qui joue sur les effets de saillance que les études visuelles mettent en avant désormais. Alors que se passe-t-il à Arromanches au musée du Débarquement pour les visiteurs ? Que pouvons-nous dire cette visite et ce dans la perspective d'un nouvel espace muséal ? ${ }^{1}$

\section{Le projet d'étude : étapes de l'observation}

Après une rencontre avec la direction du musée, il a été décidé de mettre en place une grille d'observation des visiteurs dans l'espace scénographique. Une première grille fut construite puis modifiée après une observation test menée par l'ensemble des étudiants en médiation culturelle de l'ESPE. Les outils présentés ici ont donc fait l'objet d'une expérimentation en amont et de modifications pour répondre au mieux aux conditions du musée. Le schéma ci-dessous présente ce que donne l'observation en termes de données collectées. 80 visiteurs ont ainsi pu être observés lors de ces trois mois. 


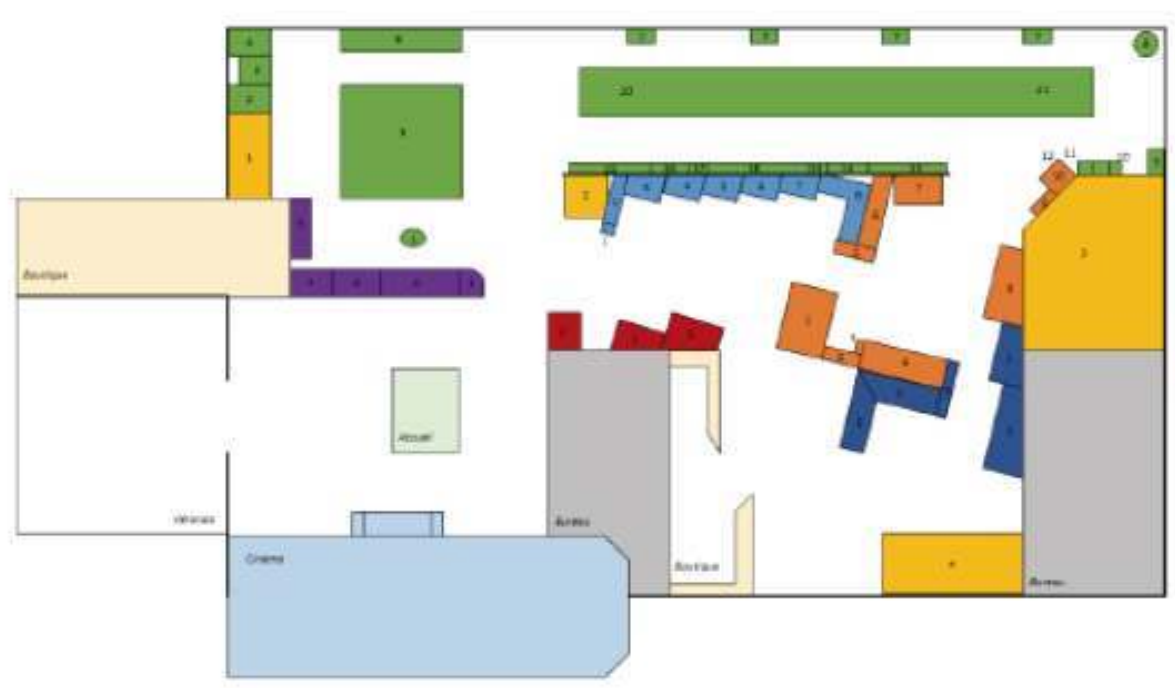

\section{L'espace observé}

5 L'espace du musée du Débarquement d'Arromanches est organisé en six zones déterminées :

6 - Avant l'assaut (en violet sur le plan) : plusieurs vitrines reprennent la vie des populations et les opérations militaires effectuées avant le débarquement ;

7 - Le port artificiel (en vert sur le plan) : maquettes du port artificiel d'Arromanches, le Mulberry B, accompagnées d'éléments de compréhension;

8 - La France libre (en orange sur le plan) : trois focus sur les forces navales et aériennes françaises ainsi que sur le commandant Kieffer ;

9 - Les alliés (en bleu sur le plan) : zone consacrée aux différents pays alliés intervenants dans la Seconde Guerre mondiale. ;

10 - L'empire Britannique (en rouge et bleu sur le plan): plusieurs vitrines destinées notamment aux divisions militaires britanniques et aux plages Gold et Sword ;

11 - Le débarquement (en orange et rouge sur le plan) : espace rassemblant, entre autres, des vitrines sur les plages d'Ohama et d'Utah.

12 Le musée est équipé de maquettes et d'un diorama : le débarquement des troupes britanniques, une réplique de péniche LCA, un diorama avec son et lumière et une scène de vie militaire.

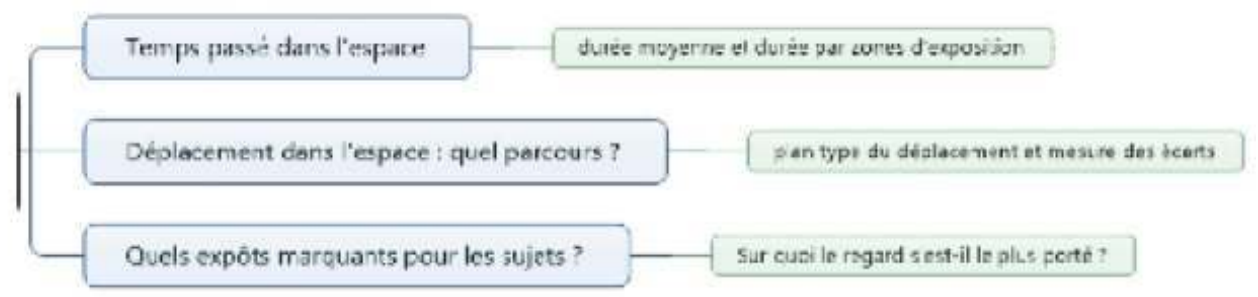


13 Pour mener l'étude, une grille d'observation est construite à partir d'une première observation dans l'espace muséal avec:

14 - un numéro attribué à chaque visiteur observé ;

15 - le contexte de visite, c'est-à-dire l'affluence de visiteurs dans le musée ;

16 - l'âge et le sexe de l'observé(e);

17 - les remarques, si le visiteur est seul ou accompagné ainsi que les éventuelles remarques concernant les visites guidées ;

18 - le parcours, les arrêts sont numérotés dans l'ordre de la visite de l'observé(e) ;

19 - les objets, c'est-à-dire le répertoire des expôts présents dans le musée, tous numérotés en

fonction de la zone dans laquelle ils se trouvent - chaque zone est définie par une couleur ;

20 - l'identification du comportement de l'observé(e) : lecture des textes explicatif ou introductif, suivi du regard (jette un œil, regard périphérique, regard global, regard détaillé, regard dévié), éventuelles discussions, photographie ;

21 - les notes : comportement autre que ceux indiqués, suivi ou non d'une visite guidée ;

22 - le temps : par expôt.

23 La grille d'observation est alors divisée selon les zones d'exposition. Un questionnaire de visite est proposé aux visiteurs observés, à chaque fin de visite. Il est composé de quatre questions :

24 - Pourquoi avez-vous choisi de visiter ce musée ? Êtes-vous déjà venu?

25 - Lors de votre visite, vous êtes-vous facilement repéré dans l'espace?

26 - Êtes-vous allés sur les plages avant de visiter ce musée ? Allez-vous vous y rendre après ?

27 - Quels sont les éléments qui vous ont le plus intéressé ? 
Les visiteurs autour de la maquette du port artificiel

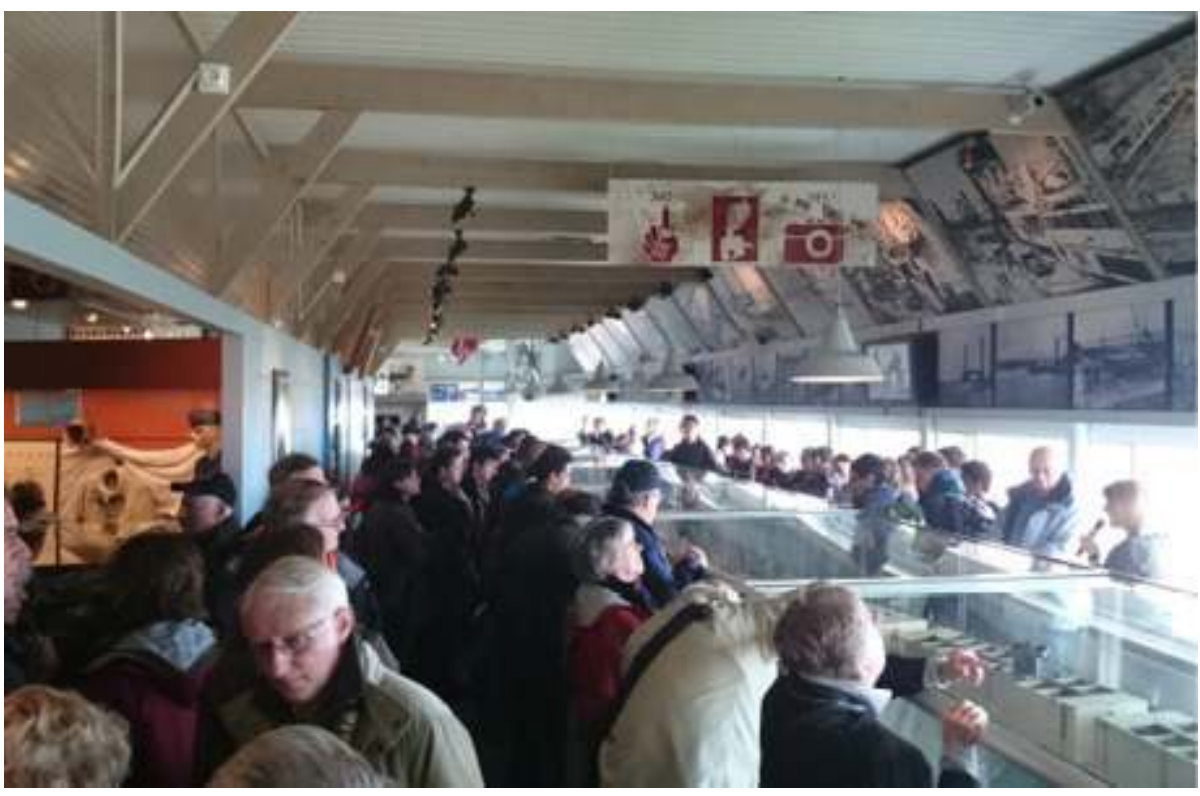

(c) Maeva Hardelay

28 Avec la grille d'observation et à l'aide des temps indiqués, un expôt marquant par zone a été identifié pour chaque observé. L'intérêt du questionnaire est alors de croiser les données des deux outils. L'expôt marquant relevé au temps est-il le même que celui relevé dans le questionnaire?

Le questionnaire permet également de mesurer le rapport entre le musée et la plage : les visiteurs y sont-ils allés avant et ont-ils trouvé des réponses à leurs questions dans le musée ? Y sont-ils allés après avoir eu les informations nécessaires à la compréhension de ce site?

Enfin, le questionnaire permet également d'indiquer à la fois les points positifs et les points négatifs du musée mais tout autant les améliorations à prévoir, les modifications et/ou changements nécessaires. Sur l'ensemble des 80 personnes observées, seules 5 ont refusé de répondre aux questions.

\section{Les conclusions de l'étude}

\section{Les résultats de l'observation}

À partir du plan de l'espace constitué, les parcours de chaque enquêté ont été reportés. Il est difficile de définir un déplacement type tant le trajet de chacun est différent du fait de la disposition même de l'espace muséal. Premier musée lié à l'histoire du Débarquement (1954), son exposition a été montée sans scénographie et aujourd'hui encore seule la présence de guides expliquant pendant une trentaine de minutes le port artificiel d'Arromanches, offre une structuration de la visite au touriste.

Nous notons que $85 \%$ des enquêtés ont regardé les vitrines de la zone 1 traitant de l'occupation allemande et de la préparation du débarquement en raison de sa proximité avec la zone d'entrée, ce qui explique ce taux conséquent. Proche de l'entrée et du départ de la visite guidée, le visiteur regarde ce qui se trouve dans cet espace. La totalité de nos 
80 visiteurs a visité la zone 2 sur le port artificiel d'Arromanches. C'est le point fort de la visite, ils viennent chercher, avant toute chose, des informations relatives au port artificiel dont les traces, vestiges sont visibles dès l'arrivée sur la commune. Les trois vitrines sur la France libre de la zone 3, ont attiré 80 \% des enquêtés. Seuls $13 \%$ ne sont pas allés visiter la zone 4 sur les alliés ayant contribué au Débarquement.

Si la fréquentation des zones d'exposition est une réalité, il est à noter que le regard porté par les visiteurs est alors davantage périphérique, allant de vitrine en vitrine, cherchant les informations essentielles à l'avance et rapidement. La quasi-totalité des enquêtés ont regardé les nombreux expôts de la zone 5 sur l'empire britannique. 82 \% ont regardé le film d'archive sur la construction du port artificiel d'Arromanches et $90 \%$ ont suivi la visite guidée, proposée par la structure muséale, apportant des éléments de compréhension sur le port artificiel d'Arromanches.

Le temps moyen de visite est de 1 heure et 5 minutes, ce qui se rapproche du temps préconisé par la structure culturelle, à savoir 1 heure et 15 minutes.

Temps moyen par zone de l'espace de visite

\begin{tabular}{|c|c|c|c|c|c|}
\hline zone 1 & zone 2 & zone 3 & zone 4 & zone 5 & zone 6 \\
\hline $1 \mathrm{~min} .43$ & $11 \mathrm{~min} .11$ & $53 \mathrm{sec}$. & $1 \mathrm{~min} .35$ & $2 \mathrm{~min} .24$ & $2 \mathrm{~min} .27$ \\
\hline
\end{tabular}

En mettant de côté la visite guidée qui retient l'attention du visiteur sur une trentaine de minutes sur le total de son temps passé dans l'espace muséal (50\%), il ressort que la zone que nous qualifions "port artificiel" est celle qui attire le plus le visiteur.

Si nous arrivons par une observation attentive et décrite dans ce rapport d'étude à percevoir ce qui capte le plus l'attention du visiteur, il est à noter que, sauf pour la zone 2 , le temps passé en moyenne est très court, ce qui signifie que le regard périphérique s'impose : le visiteur balaye du regard la vitrine proposée à la recherche de mots clés, de titres, de textes courts et qu'il se projette ensuite sur les objets. L'absence de textes pédagogiques cadrant la visite amène ce regard périphérique large. Celui-ci est très répandu dans les musées d'histoire et ne signifie pas une réelle appropriation par le visiteur. Le visiteur passe la moitié de son temps dans une visite guidée à écouter les explications liées au port artificiel d'Arromanches et ce dans un espace contraint (tout le monde ne peut voir la maquette qui est au centre de la présentation) puis à la fin de cette présentation, se retrouve seul dans le reste de l'espace muséal.

37 Les dioramas et maquettes captent à plusieurs reprises le regard du visiteur, car ils semblent offrir une explication probable au visiteur qui sort de trente minutes d'explication historique.

Enfin les maquettes du port, parce qu'elles sont intégrées à la visite guidée, font l'objet de toute l'attention du visiteur. Les réponses au questionnaire La définition de ce qui a marqué la visite se fait de deux manières. Dans un premier temps, l'observation menée sur place permet d'identifier ce qui a le plus retenu l'attention dans la visite dans une zone d'exposition (observation minutée). Dans un second temps, le questionnaire de sortie permet d'avoir le ressenti du visiteur. 

les 75 réponses obtenues, la visite guidée arrive de loin en tête avec 42 \% des enquêtés (soit 32 personnes sur 75) qui citent la visite guidée comme le point fort du musée. $36 \%$ mettent en avant les maquettes présentes dans l'exposition. Les vitrines d'exposition ne sont citées que dans $28 \%$ des cas ( 21 personnes) ; les films dans $17 \%$, et les dioramas dans $6 \%$ des cas.

Ainsi, il ressort que la visite guidée est bien ce qui est recherché par le visiteur (qui n'a pas le choix de toute façon car la modalité d'organisation du musée amène le visiteur à faire cette visite guidée) mais les maquettes nombreuses semblent ensuite apporter un complément au visiteur qui balaye assez vite les vitrines présentant des expôts de toute la Bataille de Normandie.

Êtes-vous allés sur la plage avant la visite $? 80 \%$ des visiteurs suivis se sont rendus sur la plage avant de rentrer dans le musée. Notons que la période de l'enquête (de mars à juin 2015) est aussi favorable à une découverte-promenade sur la plage.

Êtes-vous déjà venus au musée (sur le pourquoi de la visite, ils apportent tous une même réponse, la volonté de découvrir davantage de choses sur le sujet) ? $40 \%$ des visiteurs suivis déclarent connaître le musée et l'avoir déjà vu. Un musée que l'on connaît par la famille, des connaissances amicales.

Lors de votre visite, vous êtes-vous facilement repéré dans l'espace ? $59 \%$ des visiteurs répondent oui et $41 \%$ non.

\section{Des axes de réflexion pour un musée en évolution}

$46 \mathrm{Au}$ vu des observations menées et des questions posées au panel de visiteurs, nous pouvons déterminer quatre axes de réflexion.

\section{Mettre en avant l'origine du musée, son histoire}

Nous voyons avant tout, dans un musée, une collection d'objets, exposée pour permettre la rencontre avec un public. Mais, il s'agit de bien plus que cela. En effet, le musée constitue un réel discours, un langage social qui révèle une intention. Véritable expression sociale d'une intention politique, le musée se doit de donner des clés de lecture à ses visiteurs. Le musée du Débarquement d'Arromanches a la chance de s'inscrire dans une histoire forte. Ainsi, ce qui intéresse le visiteur n'est proposé qu'au travers des propos tenus par les guides ${ }^{2}$, or il apparaitt indispensable de donner une place dans une nouvelle scénographie à des questionnements nécessaires ${ }^{3}$ : pourquoi un musée ici ? Qui est à l'origine du musée ? L'observation menée et les courts entretiens montrent que ces deux questions sont importantes pour le visiteur qui a cherché des réponses sur ces terrains là d'abord et avant tout. Le taux assez conséquent de visiteurs (sur le panel de 80) qui déclare avoir une connaissance préalable du musée (par connaissance, par une visite antérieure) montre que le musée dispose d'une réputation qui précède la visite. On y vient pour les vestiges sur la plage et les maquettes, on y vient parce que le musée constitue une "étape" à vivre dans une "tradition familiale ou amicale". 
Maquette reconstituant un épisode du Débarquement.

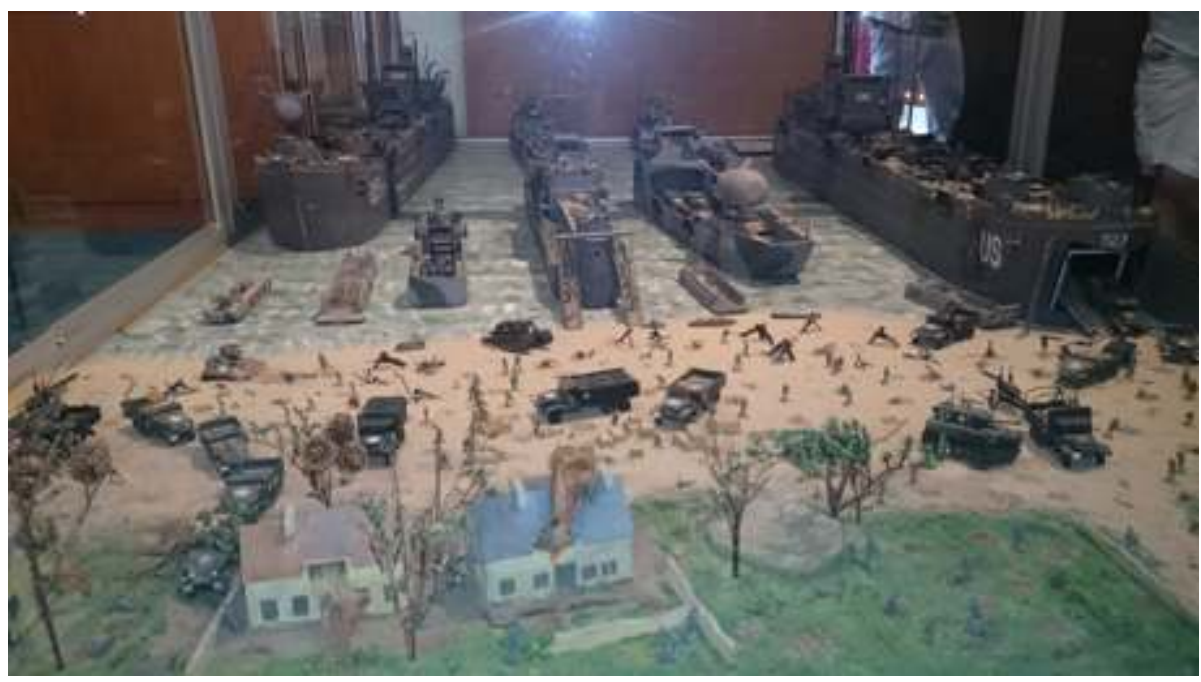

(c) Maeva Hardelay

Vitrine présentant des objets divers liés à la Bataille de Normandie.

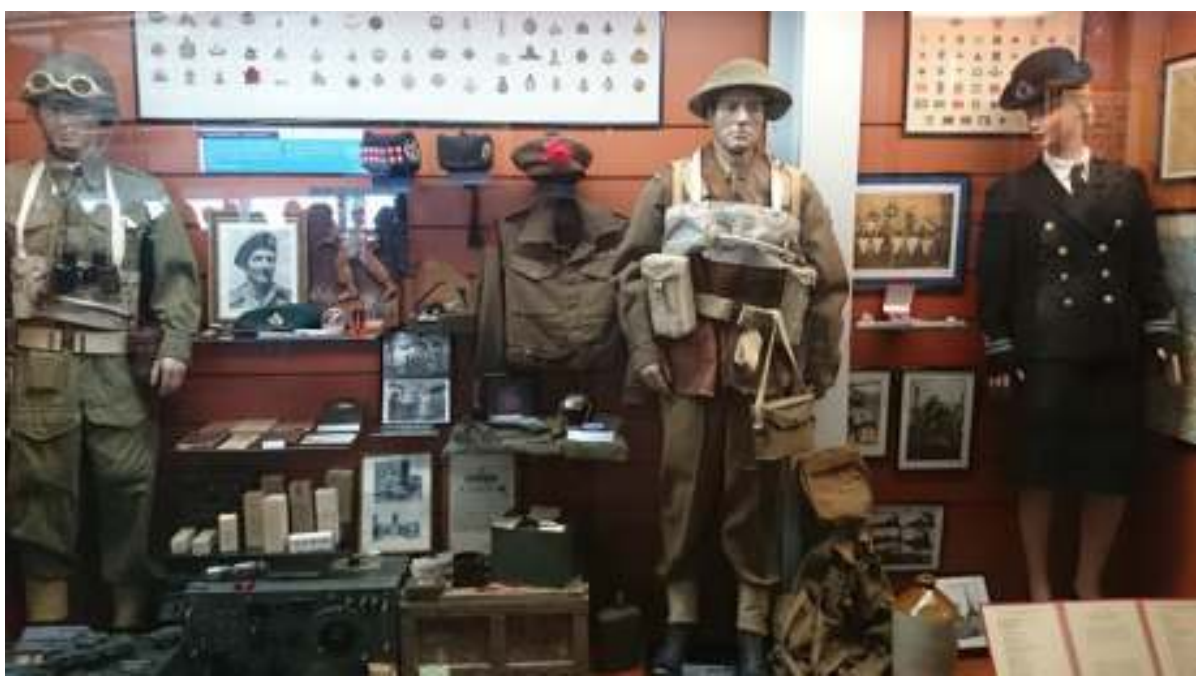

(c) Maeva Hardelay

\section{Travailler l'origine des expôts}

L'ensemble des expôts d'un musée s'inscrit dans une muséographie que l'on qualifie d'idées lorsque l'exposition elle-même donne du sens aux objets : le musée fournit alors au visiteur le mode d'emploi de l'observation. En revanche, dans le cas d'une muséographie d'objets, le sens de l'exposition provient des objets signifiants. Le visiteur utilise alors son expérience de visite et mobilise ses connaissances pour situer, comparer et donner du sens à ce qu'il observe. Aujourd'hui, face à de nouvelles possibilités d'exposer, le musée d'Arromanches doit se poser la question de l'immersion : un espace muséal immergeant le visiteur dans l'exposition avec des dispositifs interactifs innovants : comprendre la fabrication du port artificiel, son transport et son utilité sont les informations attendues des visiteurs. La focale glisse alors de l'objet au visiteur lui-même, 
un visiteur plus sensible au récit, venu non plus seulement pour les expôts mais pour lire, écouter, voir un discours véridique à travers un véritable travail de médiation lui donnant les clés de lecture d'un site et d'une histoire. Le visiteur prend sa place, il devient non plus spectateur, mais acteur d'une visite qu'il mène, choisit selon ses expériences, ses connaissances et ses envies.

Au musée du Débarquement d'Arromanches, l'empressement des visiteurs pour suivre la visite guidée montre à quel point, la recherche d'un récit prime sur la découverte des objets. Ainsi, si nous prenons en considération ce comportement des visiteurs, il nous semble essentiel de mieux valoriser l'histoire des collections. En effet, le musée d'Arromanches a été édifié à l'initiative de Raymond Triboulet, premier sous-préfet de la France libérée et l'exposition permanente du Débarquement à Arromanches a été inaugurée officiellement le 5 juin 1954 par René Coty, Président de la République.

Il s'agit du premier musée construit pour commémorer le 6 juin 1944 et la bataille de Normandie. En 2014, plus de 350000 personnes se sont rendues dans ce musée, troisième lieu de mémoire de la Bataille de Normandie le plus fréquenté. Il expose des collections d'objets divers, uniformes, armes, équipements divers qui sont à la fois le fruit d'une collecte, de dons de particuliers, d'anciens combattants, d'associations. Les réserves sont trop petites et le projet d'un nouveau musée au-delà de la conception d'un récit plus clair répond également au besoin de mieux stocker les objets. Le musée est confronté au stockage des collections depuis plusieurs années.

Avec ses plaques commémoratives Arromanches est aussi un lieu de mémoire fort.

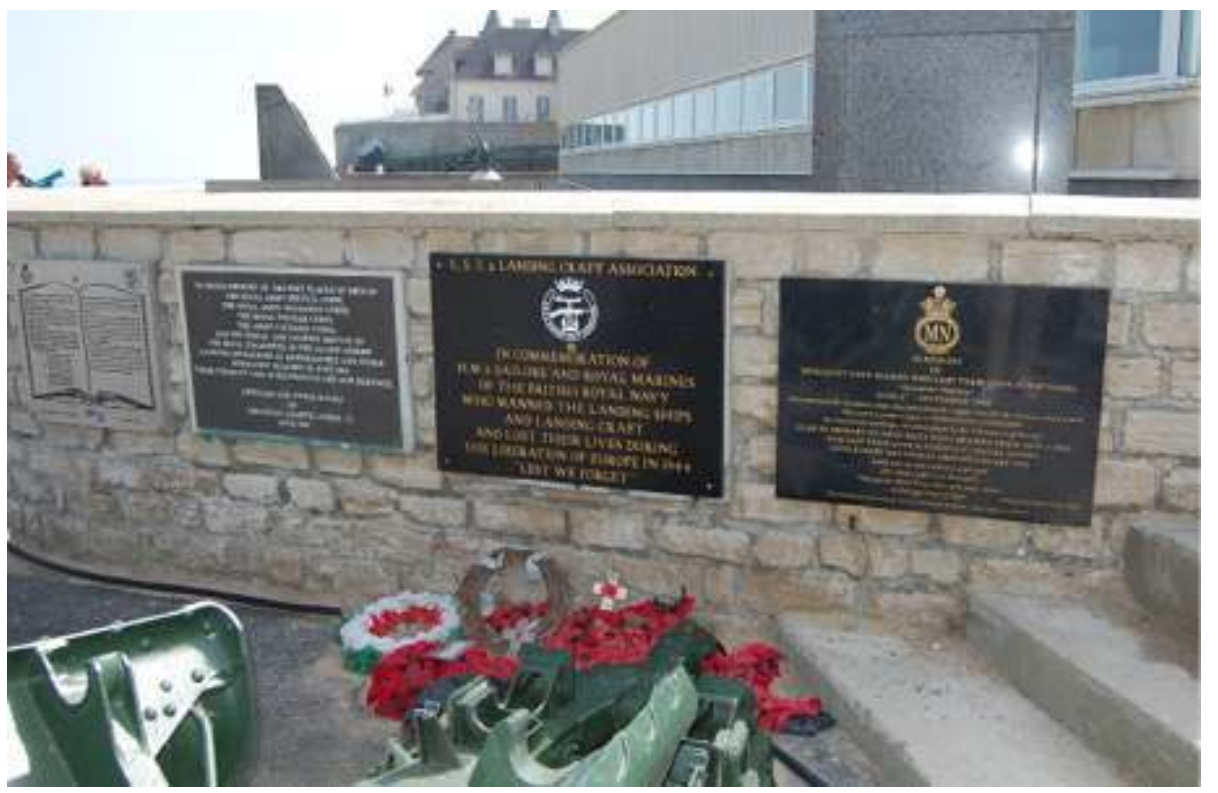

(C) S. Hommet

51 Ce musée repose sur son histoire. C'est le premier de la Normandie sur la thématique, qui a donc accumulé quantité d'expôts visibles dans d'autres espaces muséographiques désormais. Un film est encore présenté alliant vidéos et projections avec la voix de Raymond Triboulet.

52 Ce musée du Débarquement est donc spécifique, doté d'une collection conséquente et touchant tous les aspects de la Bataille de Normandie. Il apparaît nécessaire aujourd'hui de mieux y penser la place des objets dans une scénographie à concevoir : l'omniprésence 
du regard périphérique du visiteur montre à quel point, il recherche lors de sa visite ce qui lui fournit des explications dans un récit qu'il se construit. Le musée se doit de faire des choix dans les expôts présentés qui sont une collection faite au fil des années avec un lien plus ou moins fort avec le port artificiel. Travailler à une meilleure image visuelle du musée s'impose : le paysage physico-mémoriel extérieur constitue en soi une réalité. D'autre part, dans une scénographie construite et une organisation revue de l'exposition du musée, il faudra penser l'image du musée qui devrait inviter à s'immerger dans un lieu unique : au plus près des plages, là où le port fut construit, découvrir le récit de ceux qui ont permis cet exploit technique et militaire au service de la liberté. Ces recommandations s'appuient sur la nécessité de passer d'une histoire matérialisée par des objets signifiants à une histoire sentie voire expérimentée par le visiteur par le biais de médiations adaptées. Le visiteur vient de moins en moins au musée pour voir des objets qui lui sont étrangers et extérieurs par le temps et l'espace, mais pour participer à une rencontre produite par des objets authentiques, reproduits, reconstitués, dans le but de vivre une expérience unique et de s'interroger.

\section{Recentrer le récit sur le port artificiel : de la nécessité d'une scénographie}

Le musée d'Arromanches a pour nom, musée du Débarquement, et pourrait davantage mettre l'accent sur l'histoire du port artificiel. Or, le musée et son espace scénographique devraient se centrer sur celui-ci et son histoire dans le débarquement. Une scénographie qui reposerait sur un récit chrono-thématique aurait l'avantage de répondre aux attentes des visiteurs : la fabrication du port, le transport jusqu'à la plage, le fonctionnement du port et son exploitation, la tempête, le bilan (ce que le port a apporté au débarquement). Les éléments liés aux plages du débarquement, la contextualisation, la cartographie seraient à insérer dans un tel récit. L'observation menée montre à quel point, le visiteur après avoir suivi la visite guidée, balaye rapidement les vitrines composées d'objets. La richesse de la collection aurait toute sa place dans une mise en scène différente. Recentrer l'exposition dans un récit sur le port et en lien avec l'histoire du débarquement, tel devrait être l'objectif d'une nouvelle scénographie qui devrait prendre en considération l'attitude du visiteur dans l'espace d'exposition : le visiteur établit sa visite, par ses choix, attitudes, en cela il est "objet muséal" et fait preuve d'expérience de visite. 
La place centrale d'Arromanches sur laquelle cette pièce d'artillerie fonctionne comme un objet d'appel pour le musée du Débarquement.

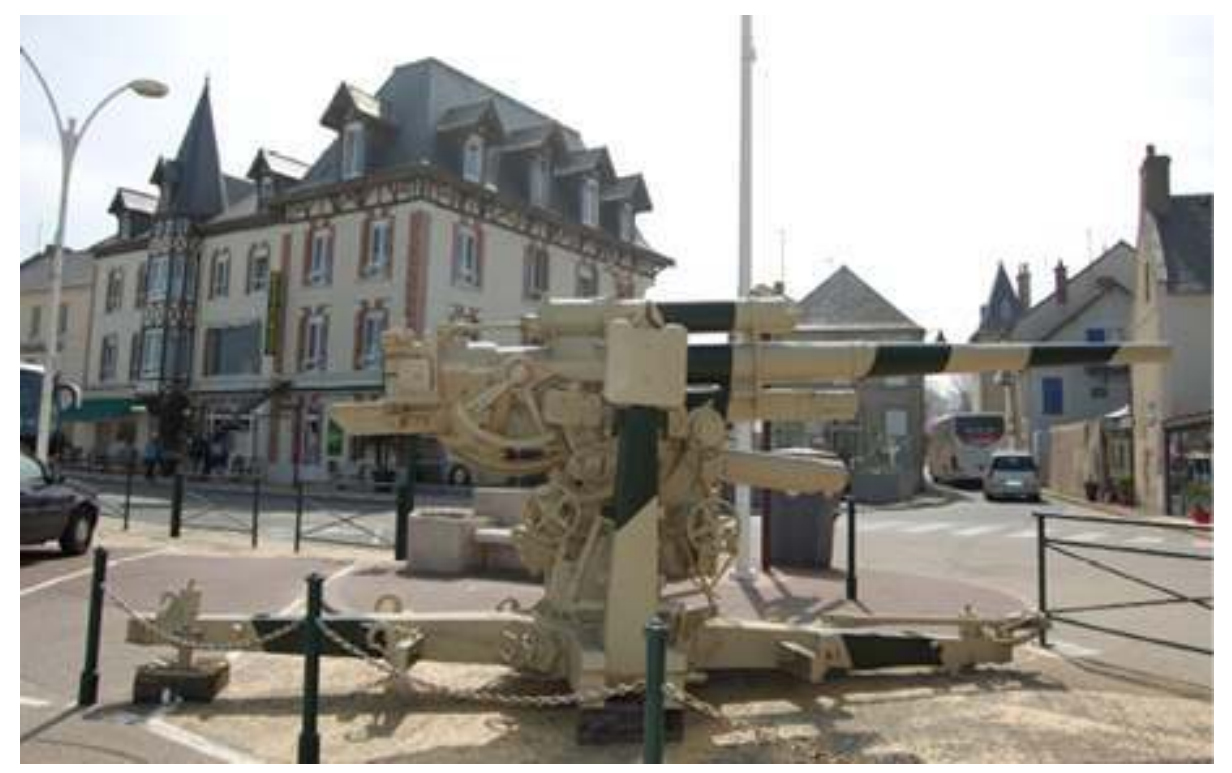

(c) S. Hommet

\section{Dans une médiation repensée}

La visite guidée proposée au sein du musée est un réel atout et un point fort. Cependant, dans le cadre d'une scénographie à concevoir qui devrait laisser davantage de place à l'expérience du visiteur, le guidage traditionnel proposé devrait évoluer vers une médiation plus adaptée avec une présence dans l'espace de médiateurs identifiables par le visiteur et fournissant des explications sur des points précis qui nécessitent un accompagnement du visiteur. La non organisation systématique de visites guidées devrait permettre de proposer des ateliers pour le public enfant et scolaire au vu de la difficile appropriation de la thématique de l'objet exposé : un port artificiel conçu pour la première fois au monde pour mener une opération militaire d'une telle ampleur. Il nous semble aussi important de penser à une médiation à l'extérieur du musée où les traces sont visibles tant sur la plage que sur la digue, sans oublier la place centrale de la commune où des expôts sont présentés comme de simples objets d'appel. Dans cette scénographie à concevoir qui laisserait une place à la médiation, les maquettes auraient un rôle central à jouer. Si, actuellement, les écrans de télévision placés et les images utilisées sont de très grande qualité (l'efficacité pédagogique est totale), la grande maquette du port constitue toujours un atout. Il apparaît nécessaire, par un jeu scénographique simple, de la mettre en valeur dans un nouvel espace. Enfin, la place des films est à repenser avec un film plus récent à faire, des petits films ou des insertions vidéo sur des écrans dispersés dans l'espace.

En faisant appel à l'École Supérieure du Professorat et de l'Éducation de l'académie de Caen pour mener une étude de réception par les publics de l'espace muséal d'Arromanches, la région Basse-Normandie cherche à faire évoluer les responsables des structures muséales et ainsi à donner toute sa place légitime à la médiation dès l'origine et la conception des espaces d'exposition. C'est à ce titre aussi que le classement des 
plages du Débarquement au patrimoine mondial de l'Unesco pourra revêtir un intérêt réel pour nos publics futurs.

\section{BIBLIOGRAPHY}

Chaumier, S. La médiation culturelle. Paris : Armand Colin, 2013, 284 p.

Daignault, L. L'évaluation muséale. Québec. PUQ, 2011, 348 p.

Daignault, L. et Schiele, B. (dir.) Dépasser ou réinventer l'évaluation, in Les musées et leurs publics, savoirs et enjeux. Québec: PUQ, 2014, pp. 71-83.

Hommet, S. Rapport d'étude sur le musée d'Arromanches, http://www.unicaen.fr/espe

\section{NOTES}

1. La région Basse-Normandie en lien avec la commune d'Arromanches souhaitait cette étude en amont de tout aménagement futur du musée. Notons que le projet d'un nouveau musée a été voté le 15 octobre 2015.

2. Les visites guidées ont fait l'objet également d'une observation permettant d'identifier les propos tenus : explication rapide de l'histoire du musée, origine du port artificiel, son installation et son apport dans la bataille de Normandie.

3. Le rapport d'étude sert de cadre au travail pour la nouvelle scénographie du futur musée d'Arromanches prévu pour une ouverture au public en 2019.

\section{INDEX}

Mots-clés: Réception par les publics, musée d'Histoire

\section{AUTHORS}

\section{STANISLAS HOMMET}

directeur de l'ESPE de l'académie de Caen, vice-président de l'université de Caen Normandie, responsable du parcours de Master 2 Médiation culturelle, chercheur au laboratoire ThéodileCirel de l'université de Lille 3 stanilas.hommet@unicaen.fr 


\section{OCÉANE COSTEY}

étudiante en Master 2 Médiation culturelle oceane.costey@gmail.com

\section{MAËVA HARDELAY}

étudiante en Master 2 Médiation culturelle maeva.hardelay@hotmail.fr 